
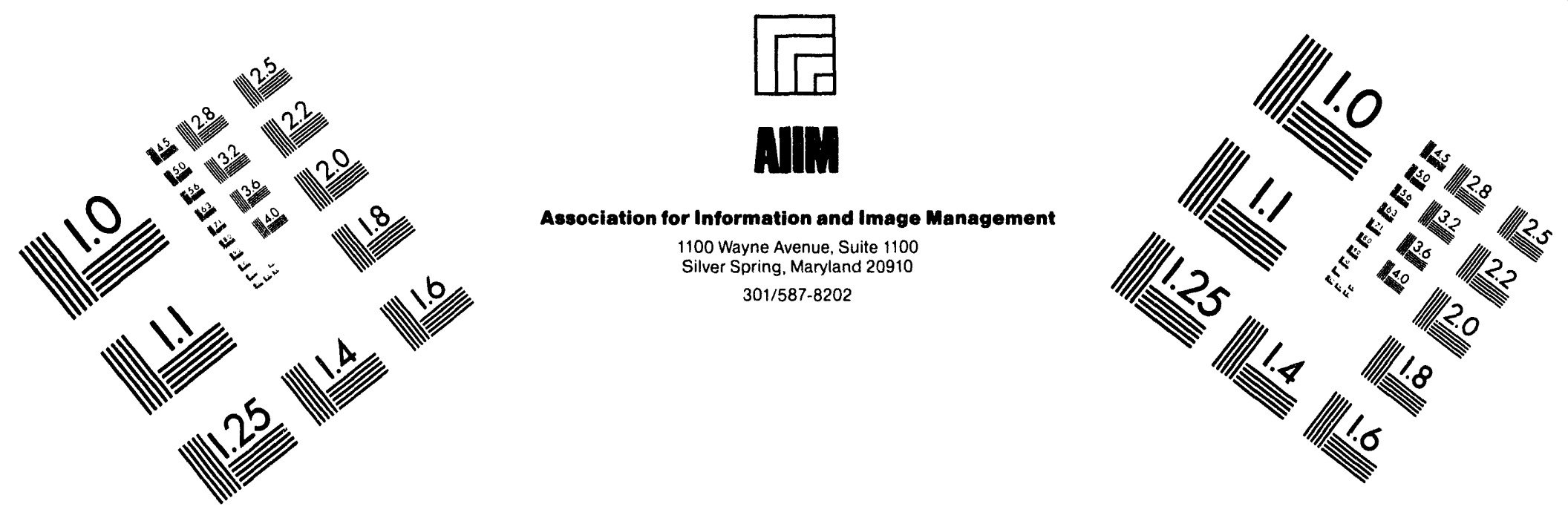

\title{
Centimeter
}

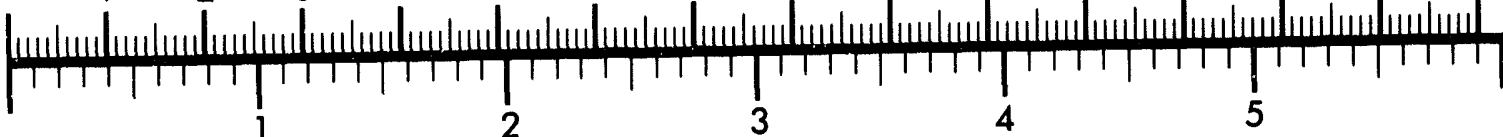
Inches
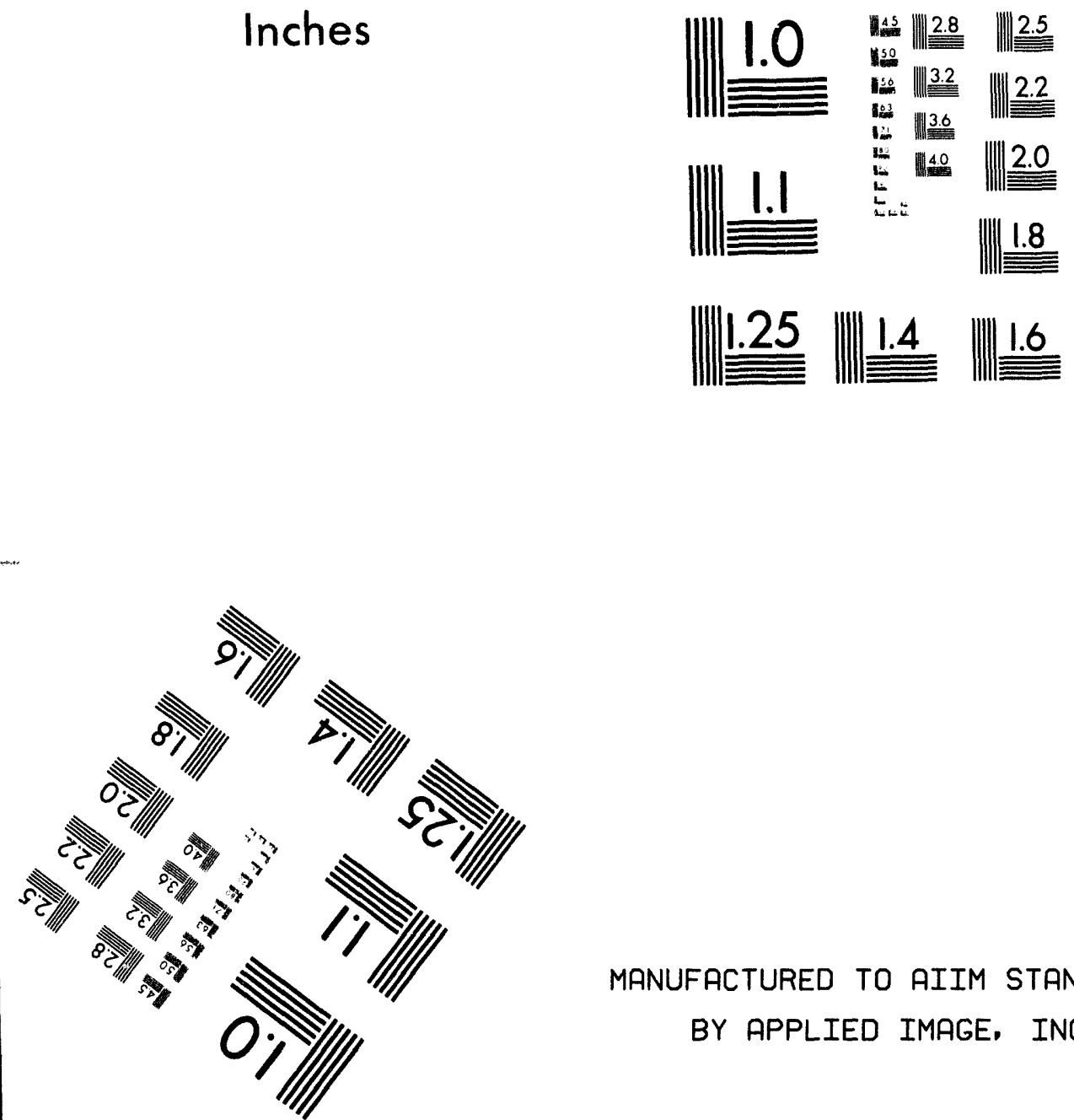

MANUFACTURED TO AIIM STANDARDS

BY APPLIED IMAGE, INC.

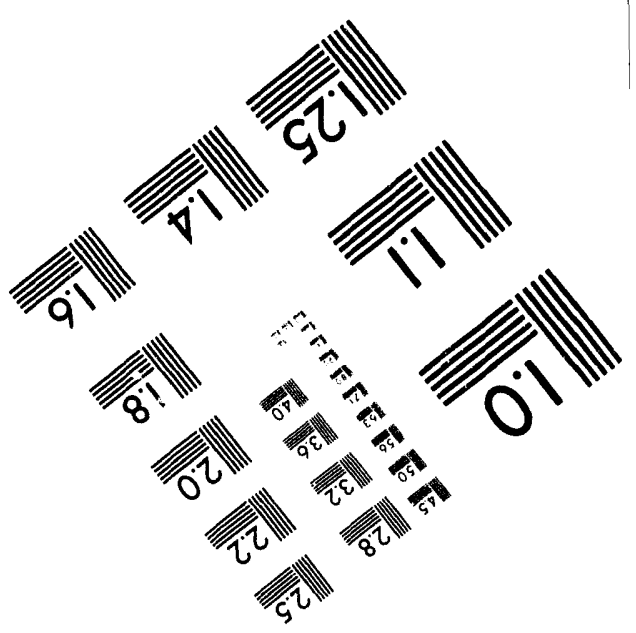



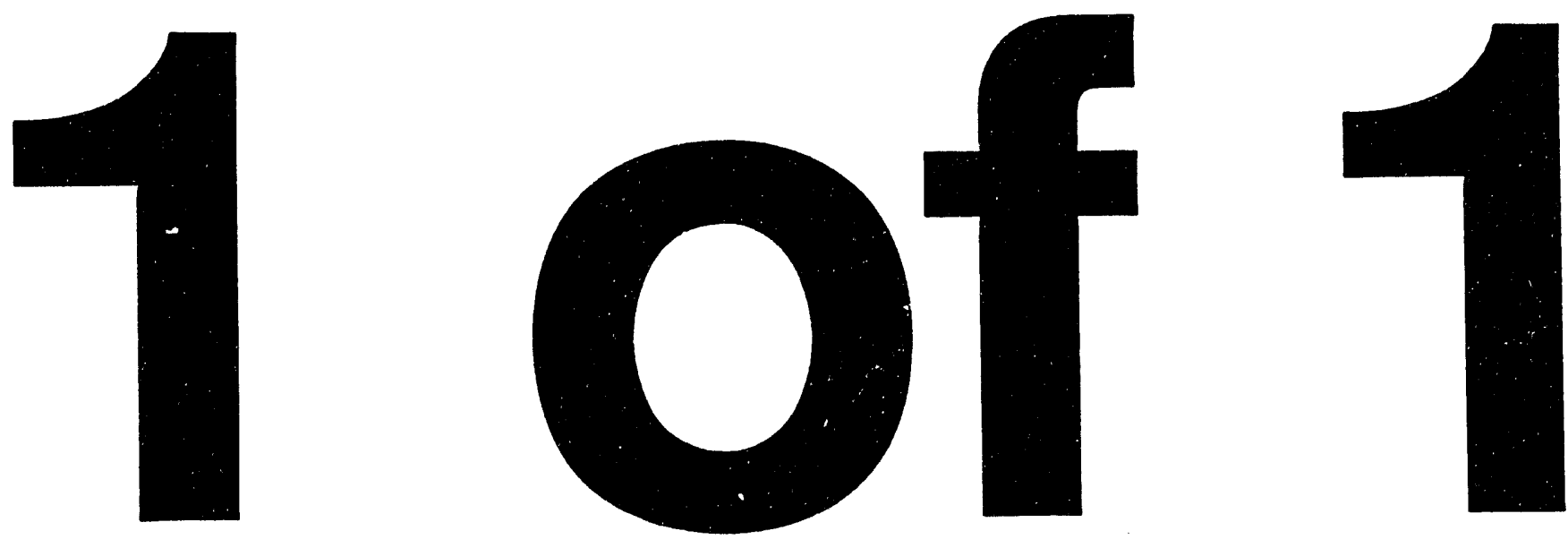
LBL-35626

\section{AEM and HREM Evaluation of Carbon Nanostructures in Silica Aerogels}

X.Y. Song*, W. Cao and A.J. Hunt

Energy \& Environment Division

Lawrence Berkeley Laboratory

University of California, Berkeley, CA 94720

*Permanent address:

Natinal Laboratory for Fine Ceramics \& Structure

Shanghai Institute of Ceramics

Chinese Academy of Sciences

Shanghai, China

Proc. Spring MRS, 4/4-4/8/94, San Francisco, CA.

To appear in Volume 349

This work was supported in part by the Director, Office of Energy Research, Office of Basic Energy Sciences, Materials Science Division of the U.S. Department of Energy under Contract No. DE-AC03-76SF00098. 


\title{
AEM AND HREM EVALUATION OF CARBON NANOSTRUCTURES IN SILICA AEROGELS
}

\author{
X.Y. SONG*, W. CAO AND A.J. HUNT \\ Energy and Environment Division, Lawrence Berkeley Laboratory \\ University of California, Berkeley, CA 94720 \\ *Permanent address: National Lab for Fine Ceramics and Structure, Shanghai \\ Institute of Ceramics, Chinese Academy of Sciences, Shanghai, China
}

\section{ABSTRACT}

Nanostructured carbon has been deposited in silica aerogels by chemical vapor infiltration using acetylene or ferrocene at moderate temperatures. Using analytical electron microscopy and high-resolution electron microscopy, we have observed various carbon rings and nanotubes in the silica aerogel-based carbon composite. Both X-ray microanalysis and nano-probe diffraction techniques have been used to confirm the presence of those carbon nanostructures. The morphologies and structural properties of the carbon nanotubes and rings have also been examined in detail.

\section{INTRODUCTION}

The discovery of novel carbon tubes of nanometer dimensions $[1,2]$ has greatly stimulated studies in the field of carbon fiber growth. Their potential applications in electronics and advanced materials have been suggested $[3,4]$. Theoretical studies predict that their electronic properties depend on the diameter of the tube and degree of helicity $[5,6]$. Much research has focused on the texture and formation processes of carbon nanostructures [7-11] Up to now, the synthesis of carbon nanotubes is based on an arc discharge method similar to that of Ebbesen and Ajayan [7]. No other methods have been reported on forming carbon nanotubes. In this paper we report the observation of not only carbon nanotubes but also nanometer-scale carbon rings and carbon fibers in silica aerogels by high-resolution electron microscopy (HREM). In addition, the carbon rings were examined and evaluated in situ using analytical electron microscopy (AEM).

\section{EXPERIMENTAL}

Silica aerogels are highly porous materials prepared using sol-gel processing and supercritical solvent extraction [12]. The first step is to mix tetraethylorthosilicate (TEOS), water, alcohol and catalyst (usually a base e.g., ammonia) in appropriate proportions. TEOS undergoes many steps of hydrolysis and condensation reactions, leading to the formation of a soft material called an alcogel. Then liquid carbon dioxide is used to replace the fluid in the alcogel and evacuated above the supercritical temperature and pressure to make aerogels. Silica aerogels have a high porosity $\left(90 \%\right.$ or higher) and surface area $\left(800-1000 \mathrm{~m}^{2} / \mathrm{g}\right)$, an ideal matrix for the chemical vapor infiltration of a second solid material on an extremely fine scale. The deposition of carbon was carried out in a tube furnace by the decomposition of acetylene at $550^{\circ} \mathrm{C}$ and post-treated in an inert atmosphere at $670^{\circ} \mathrm{C}$ for $0.5 \mathrm{~h}$. The carbon deposition was found to distribute homogeneously throughout the aerogel 
volume and the weight of the composite material increased by about $8.7 \%$ after the deposition of $1 \mathrm{~h}$. In another similar experiment, carbon and iron particles were simultaneously deposited in the aerogel by the decomposition of ferrocene at $120^{\circ} \mathrm{C}$ for $\sim 20 \mathrm{~h}$ in a helium atmosphere ( $200 \mathrm{torr})$. The as-deposited samples were further treated in an inert atmosphere at $700^{\circ} \mathrm{C}$ for about $1 \mathrm{~h}$. More detailed information on the deposition process can be found elsewhere [13]. HREM on the carbon-doped silica aerogel was performed using a JEM-200CX and TOPCON-002B operating at $200 \mathrm{KV}$. The JEM-200CX analytical electron microscopy(AEM) is equipped for energy dispersive $X$-ray spectroscopy (EDS) with an ultra-thin window germanium detector capable of detecting elements down to $C$. Both $X$-ray microanalysis and electron nano-probe diffraction techniques were used to identify the presence of carbon nanotubes and rings in the aerogel composite. Samples for HREM studies were ground to fine powders in acetone and the suspension was dipped onto copper grids covered by a holey carbon film for transmission electron microscopy (TEM).

\section{RESULTS AND DISCUSSION}

HREM observations show that some of the carbon in silica aerogels has an unusual morphology of graphite fibers and tubes. The length of carbon fibers ranges from several to hundreds of $\mathrm{nm}$ with the width varying from several to tens of graphite layers. The typical carbon nanotubes in the aerogel have a length of up to $100 \mathrm{~nm}$ and a width of $\sim 10 \mathrm{~nm}$. Figure 1 is an HREM micrograph of a typical carbon nanotube

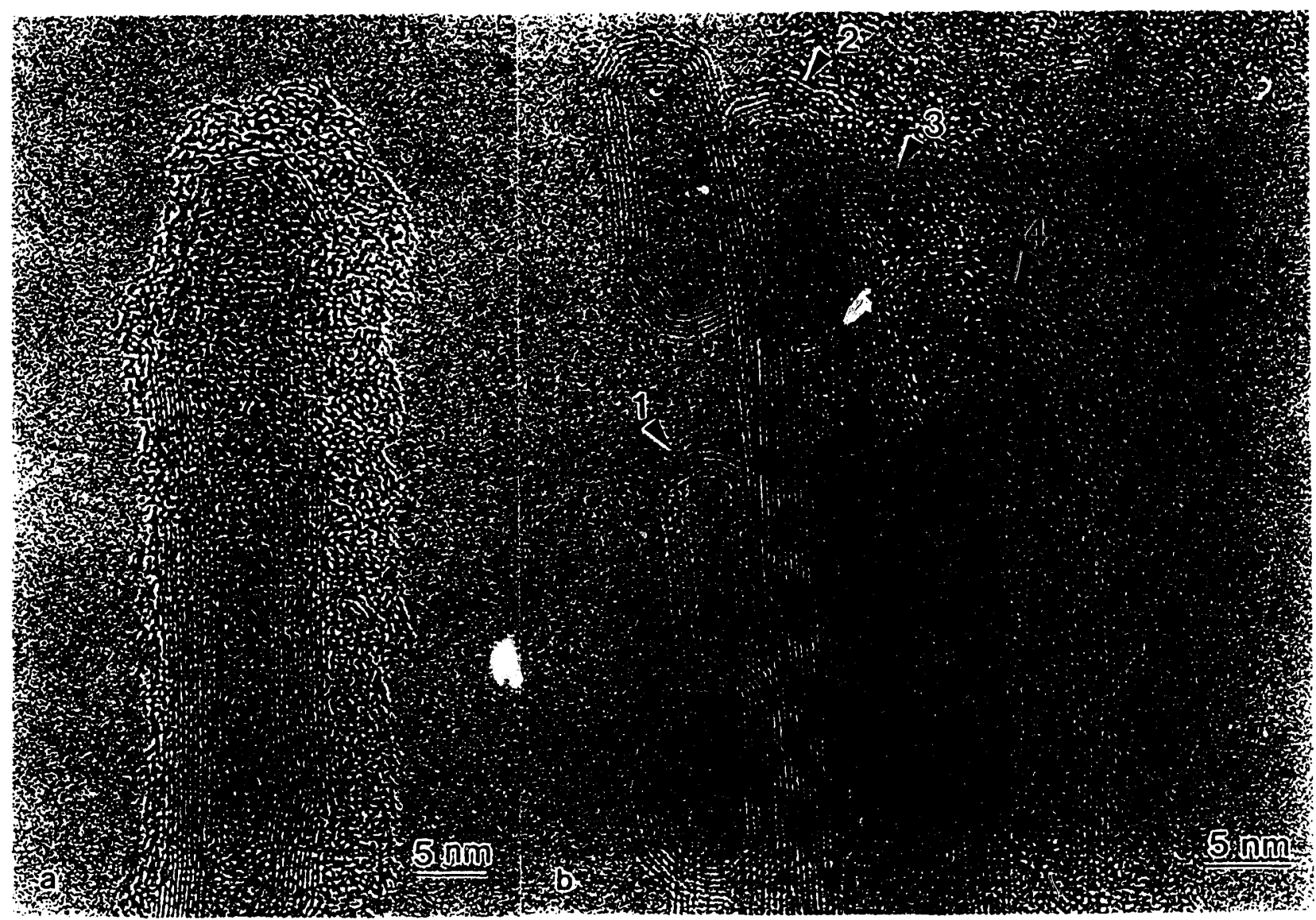

Fig. 1. HREM image showing (a) cone tip of the carbon nanotube and (b) three adjacent nanotubes parallel to each other. 
surrounded by the amorphous silica. Statistically speaking, carbon nanotubes are observed under TEM on several occasions in a 1-hour period. The tubes have $\{002\}$ lattice images of the graphite structure along the tube axes. The tube shown in Fig.1a consists of 12 symmetric graphite sheets with a spacing of $0.34 \mathrm{~nm}$ and a diameter of $\sim 12 \mathrm{~nm}$. Termination of the tube showing a cone tip is in agreement with lijima [14] who reported that the conical surface in carbon nanotubes corresponds to the presence of five pentagons at the tip surface and that the cylindrical tubes are closed usually by polyhedral caps. Three nanotubes arranged almost parallel to each other can be identified in Fig. $1 \mathrm{~b}$. There are a total of four tubes marked by the numbers 1 through 4, respectively; tubes 2 and 3 are overlapped perpendicularly but in parallel to tubes 1 and 4 . This observation is very similar to that of R.S. Ruoff [2] who discovered two nanotubes parallel to each other. In addition, two small rings or tube derivatives can be seen in Fig. 1 b.

We have also found some interesting structures as shown in Fig. 2 for a seamless graphite ring. The graphite ring has an outer diameter of about $100 \mathrm{~nm}$ and a wall thickness of $20 \mathrm{~nm}$. In order to confirm whether it is a ring or a hollow sphere, the electron nano-probe diffraction technique (minimum probe diameter 2 to $5 \mathrm{~nm}$ ) in ultramicro area was used. The results of the electron nano-probe diffraction for the ring show strong (002) spots as shown in the top inset of Fig. 2. Indexing of the diffraction pattern indicates its is a graphite structure with the strongest intensity being from (002). The nano-probe diffraction corresponding to an empty area in the center of the ring ( marked by $E$ ), however, exhibits the amorphous character as shown in the bottom inset of Fig. 2. This means that the image observed is indeed a ring and not a spherical shell structure. If there were any graphite structures in the center of the ring, the diffraction pattern would have contained strong diffraction spots. The electron micrograph shown in Fig. 3 is enlarged from that of Fig. 2. It can be seen clearly that the ring wall is composed of stacks of $\sim 60$ graphite layers (or sheets) with a spacing of $0.34 \mathrm{~nm}$. The graphite layers winding off the ring wall (marked by D) may have been caused by grinding of the TEM sample.

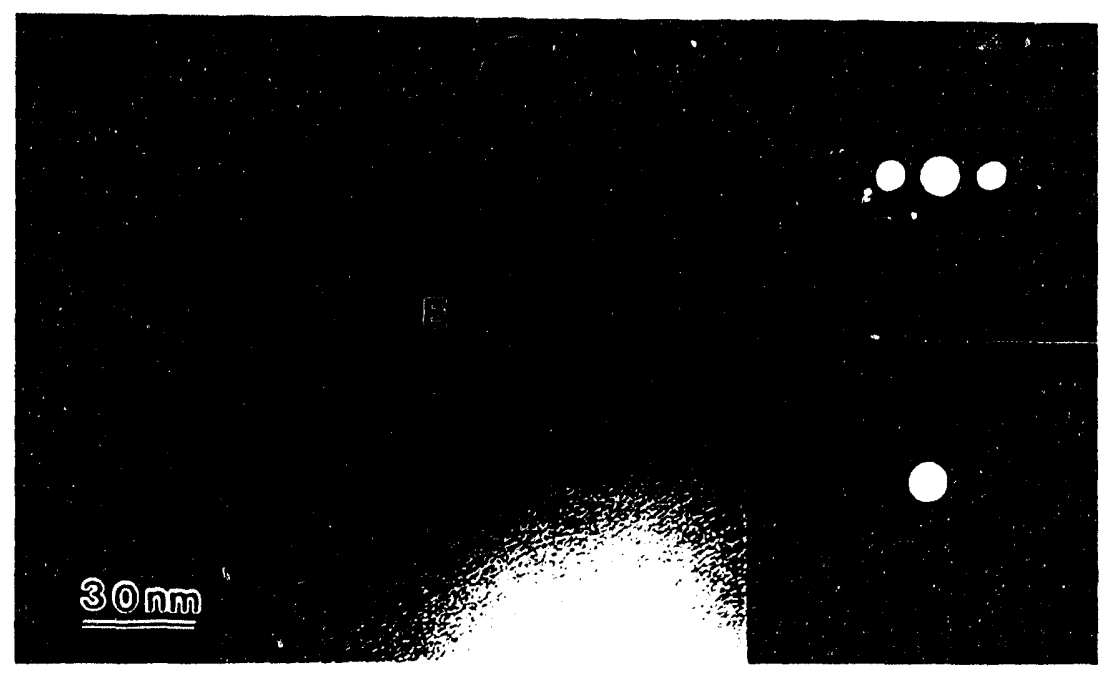

Fig. 2. TEM micrograph of a circular carton ring. The top inset is the electron nanoprobe diffraction pattern for the ring wall and the bottom inset for an area in the center of the ring. 


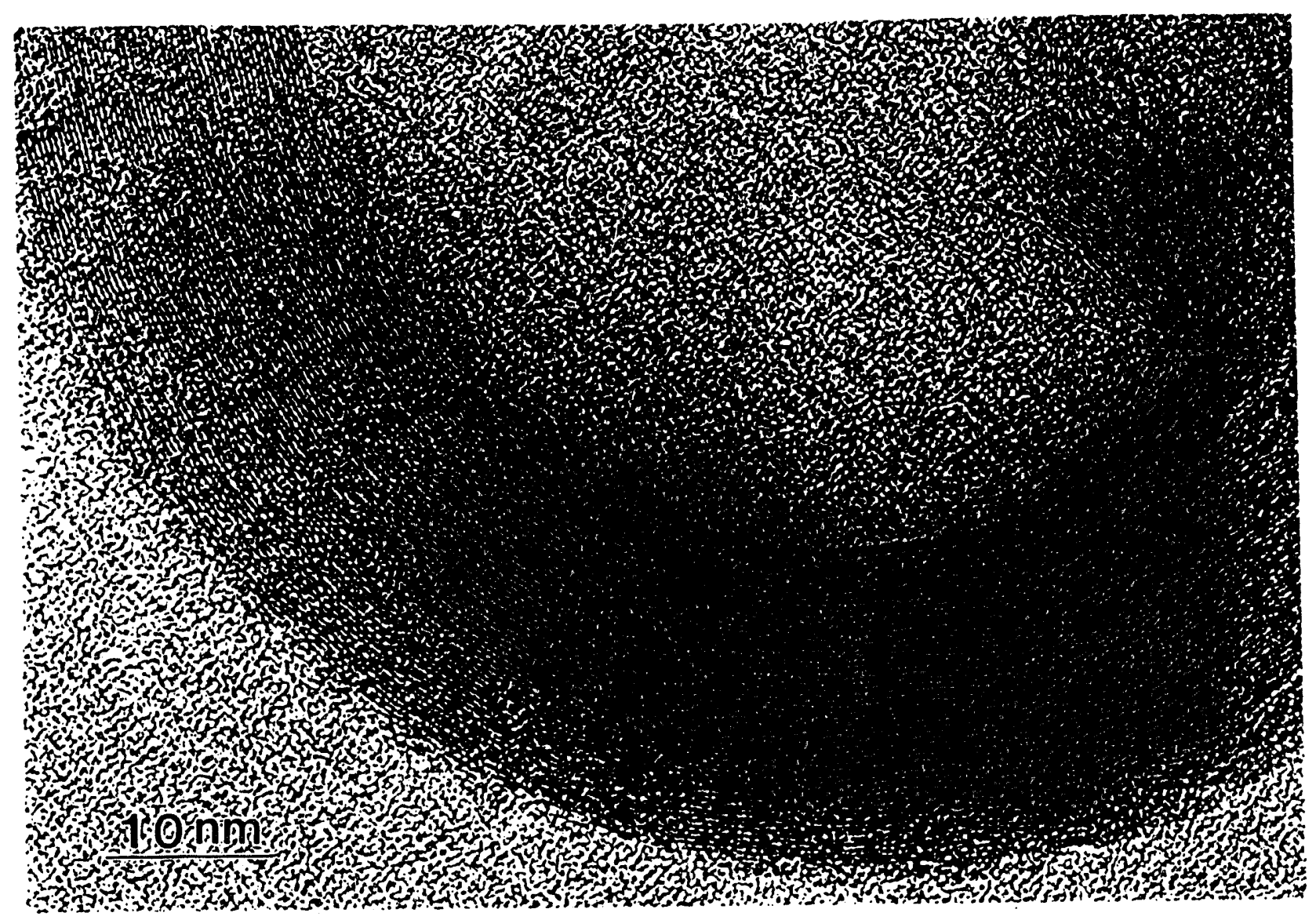

Fig. 3. HREM image enlarged from Fig.2.

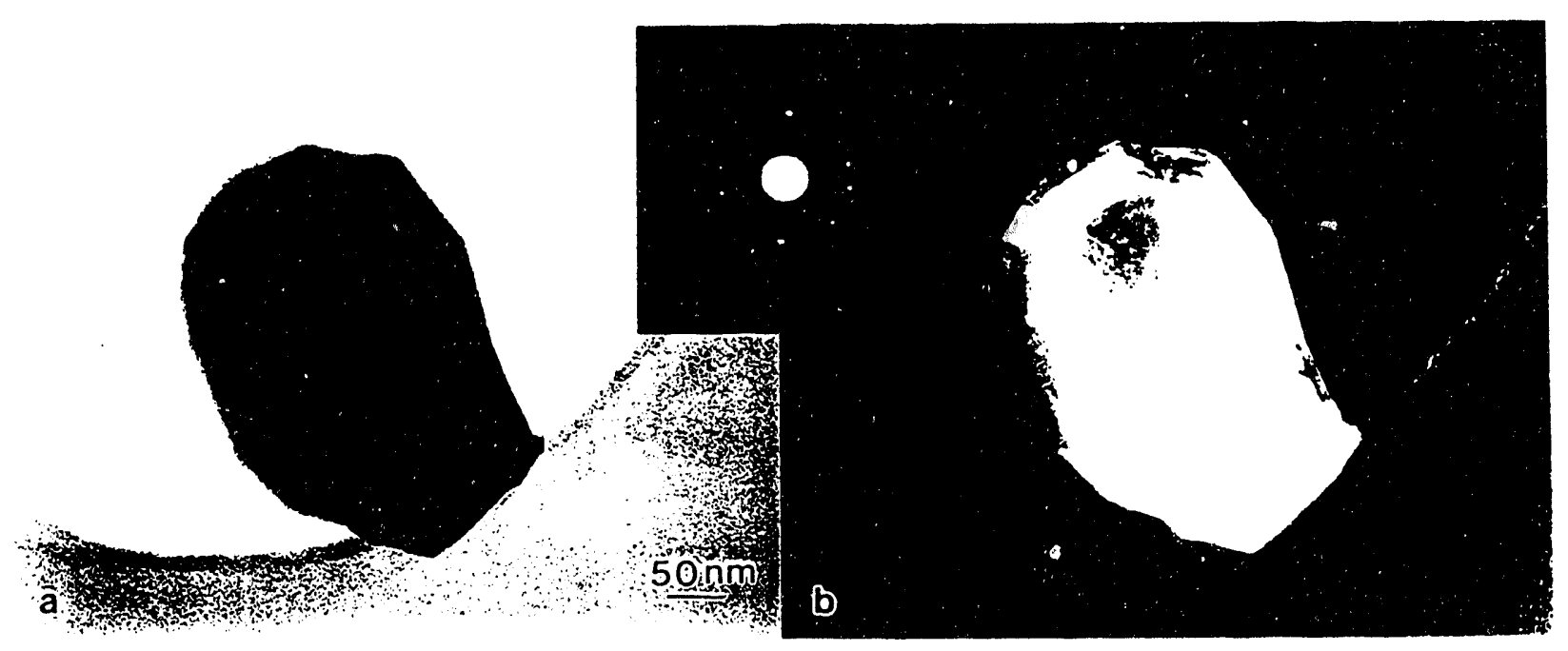

Fig. 4. TEM (a) bright and (b) dark field images of an irregularly shaped carbon ring. The inset is the corresponding SAD pattern. 

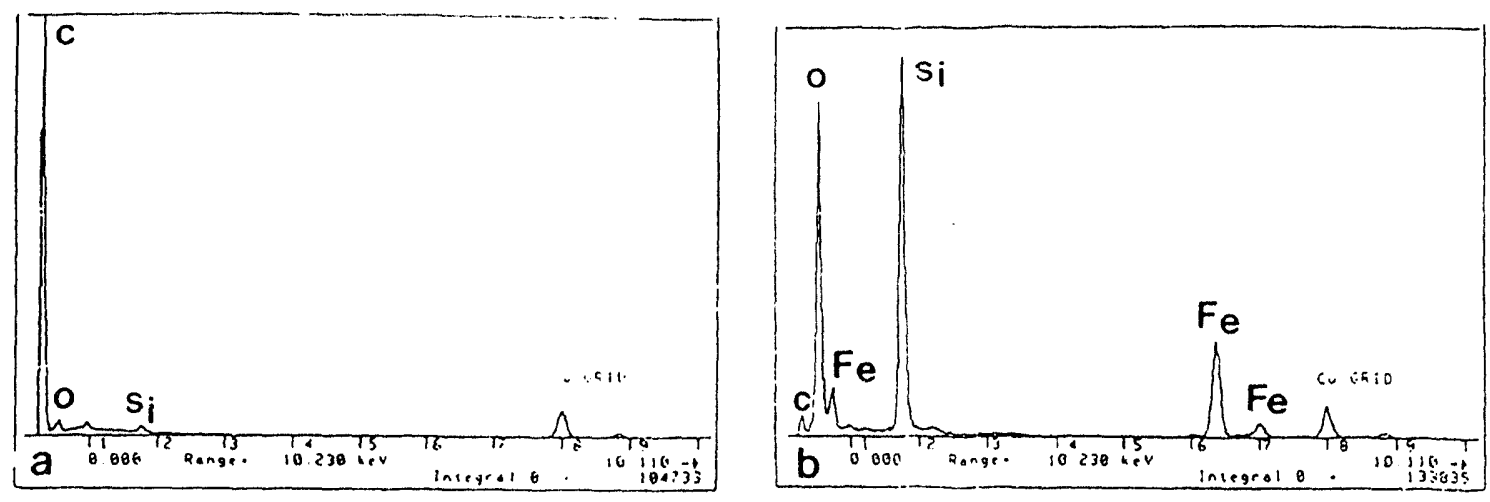

Fig. 5. EDS microanalyses of the ring structure shown in Fig. 4:

(a) the ring wall and (b) the ring center.

It is further necessary to confirm the composition of the rings present in the aerogel composite. The rings observed under TEM range from 30 to $500 \mathrm{~nm}$ in diameter. Some have an irregular shape while others appear circular much as those shown in Figs. 2 and 3. Shown in Fig. 4 are the TEM bright and dark field images of the graphite ring having an outer size up to $400 \mathrm{~nm}$ and a wall thickness of $40 \mathrm{~nm}$. The ring has a polyhedral shape which requires total 12 pentagons to be placed into hexagonal graphite sheets [15]. The result of the select area diffraction (SAD) patterns (the inset in Fig.4) shows the separate (002) spots and coincides with the ring morphology. We can also see that much of the ring except a small edge is suspended in midair without being supported by the holey carbon film on the copper grid. So we can get an accurate EDS spectrum for the ring.

Compositions for the wall and the center of the ring are identified in Fig. 5 (a) and (b), respectively, with the size of the probe being equal to approximately $20 \mathrm{~nm}$. The ring wall has a strong carbon peak, indicating it is a graphite structure (the low Cu peak comes from copper grid). The EDS spectrum for an amorphous area near the center of the ring shows a very low carbon peak but high intensities of $\mathrm{Si}, \mathrm{O}$ and $\mathrm{Fe}$, which corresponds to the silica aerogel containing some iron or iron oxide decomposed from ferrocene in the deposition process.

Our electron microscopy studies have confirmed the presence of carbon nanostructures including rings and tubes in the silica aerogel-based composite. The process is different from the arc discharge method and it remains unclear how such carbon nanostructures are formed in the silica aerogel having a mean pore diameter of $10-20 \mathrm{~nm}$. Further work is under way to identify and better control the processing parameters for the synthesis of carbon nanotubes and rings.

\section{CONCLUSION}

The presence of carbon nanotubes and other nanostructures in the aerogel composite has been identified by high-resolution electron microscopy. Using analytical electron microscopy and electron nano-probe diffraction technique, the morphologies of the ring structure have been further evaluated. The carbon ring has a structural feature composed of (002) graphite sheets with the ring center containing little carbon and being amorphous as expected. 


\section{ACKNOWLEDGMENTS}

The authors are grateful to the National Center for Electron Microscopy for providing us HREM and AEM facilities and thank Dr.U. Dahmen, C. Nelson, C. Echer, J. Turner and D.A. Tye for the help and discussions. This work were supported by both the Assistant Secretary for Conservation and Renewable Energy, Advanced Industrials Program of the Advanced Industrial Concepts Division, Office of Industrial Technologies of the U.S. Department of Energy and the Director, Office of Energy Research, Office of Basic Energy Sciences, Materials Sciences Division of the U.S. Department of Energy under contract No. DE-AC03-76SF00098.

\section{REFERENCES}

1. S. lijima, Nature 354, 56-58 (1991).

2. R.S. Ruoff, J. Tersoff, D.C. Lorents, S. Subramoney, and B. Chan, Nature 364 , 514-516 (1993).

3. P.M. Ajayan and S. lijima, Nature 361, 333-334 (1993).

4. P. Ross, Sci. Am. 265, 16 (1991).

5. J.W. Mintmire, B.I. Dunlap, and C.T. White, Phys. Rev. Lett. 68, 631-634 (1992).

6. N. Hamada, S. Sawada, and A. Oshiyama, Phys. Rev. Lett. 68, 1579-1581 (1992).

7. T.W. Ebbesen and P.M. Ajayan, Nature 358, 220-222 (1992).

8. X.P. Zhang, X.B. Zhang, C.V. Tendeloo, S. Amelinckx, M. O. Beeck, and J.V. Landuyt, J. Crys. Growth 130, 368-382 (1993).

9. J. Jiao in Proc. 51st Annual Meeting of the Microscopy Society of America (San Francisco Press, San Francisco, 1993) pp. 750-751.

10. X. Lin, X.K. Wang, V.P. Dravid, R.H. Chang, and J.B. Ketterson, Appl. Phys. Lett. $64,181-183$ (1994)

11. Y. Saito, T. Yoshikawa, M. Okuda, and N. Fujimoto, J. Appl. Phys. 75 (1) 134-137 (1994).

12. P.H. Tewari, A.J. Hunt, J.G. Lieber, and K.D. Lofftus in Aerogels, edited by J. Fricke (Springer-Verlag, Berlin, 1986) pp. 31-37.

13. W. Cao and A.J. Hunt, in this Symposium Proceedings.

14. S. lijima, T. Ichihashi \& Y. Ando, Nature 356, 776-778 (1992).

15. P.M. Ajayan, T. Ichihashi, and S. lijima, Chem. Phys. Lett. 202 (5) 384-388 (1993). 

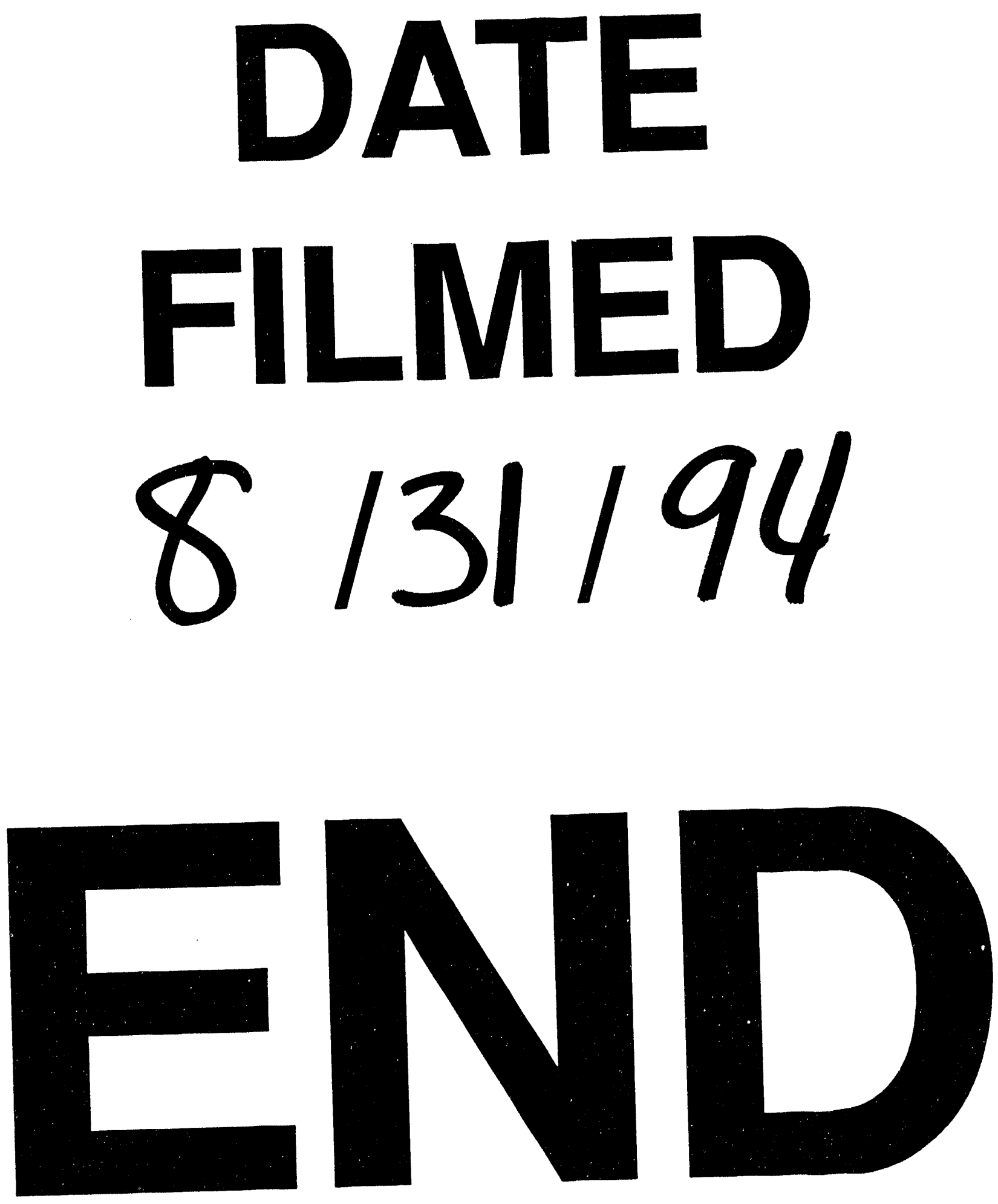
$$
\text { - }
$$
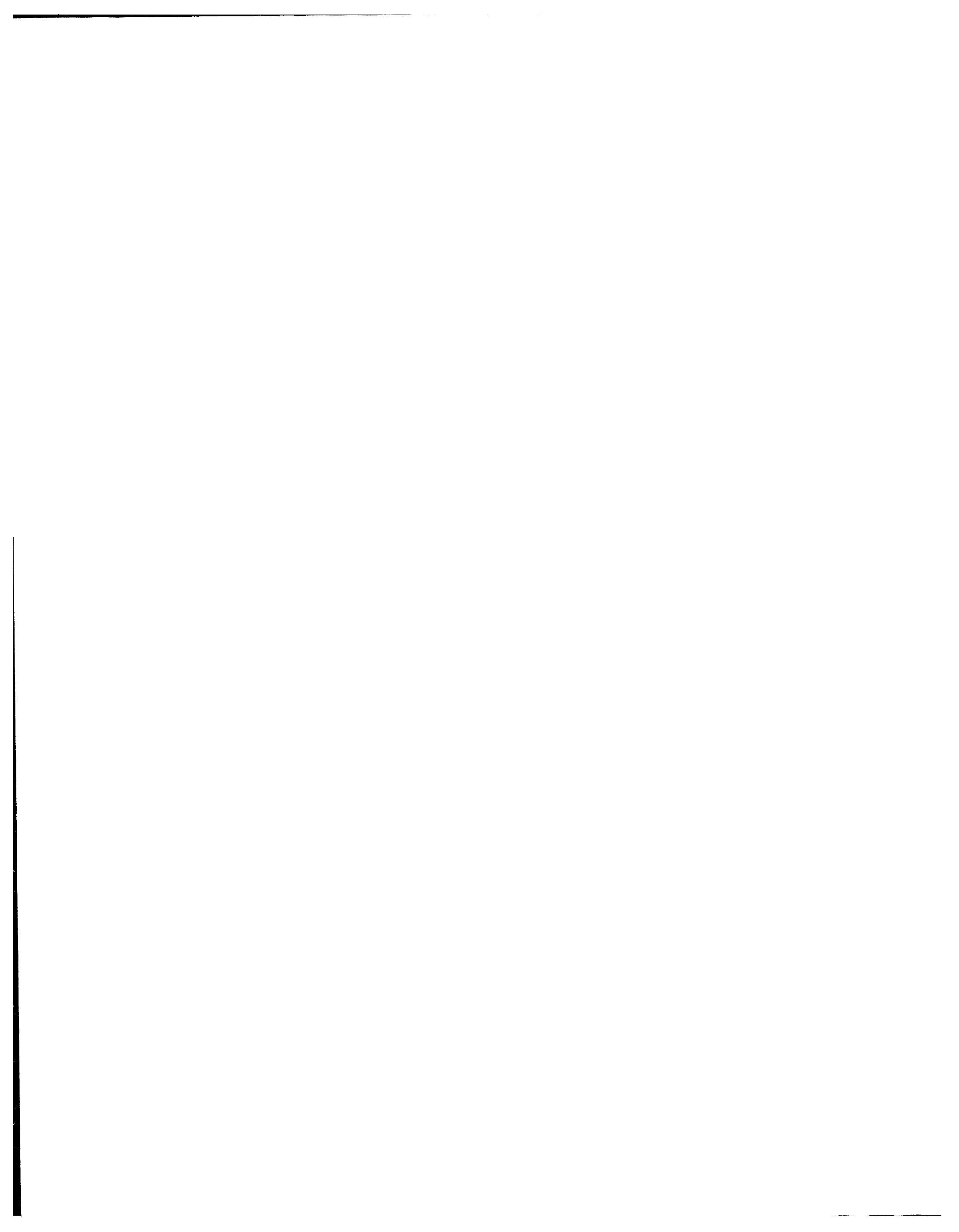